\title{
Production simulations based on a productivity potential proxy function: An application to UNISIM-I-D and PUNQ-S3 synthetic reservoirs
}

\author{
Waldir L. Roque ${ }^{1}\left[\right.$ Cristina P. Araújo ${ }^{1}$
}

Received: 3 July 2020 / Accepted: 8 October 2020 / Published online: 19 October 2020

(c) The Author(s) 2020

\begin{abstract}
Oil recovery from a reservoir involves quite a lot of challenges related to well placement before any action can be taken. An important step is proposing a reservoir target area that provides an indication of high potential for oil recovery. In other words, a position presenting high productivity potential according to some criteria. A simple proposal is the potential productivity proxy function (PP) based on the oil-bearing and mobility capacities of oil suggested as $\phi k S_{\mathrm{o}}{ }^{n}$, where $n$ is a correlation parameter, $\phi$ is porosity, $k$ is absolute permeability and $S_{\mathrm{o}}$ is oil saturation at grid block. In this paper, we consider this proxy function as a well placement strategy based on the column-wise $\left(\mathrm{PP}_{\mathrm{C}}\right)$ and layer-wise $\left(\mathrm{PP}_{\mathrm{L}}\right)$ values. To test this model, the UNISIM-I-D and PUNQ-S3 synthetic reservoirs are considered. Several production simulations are realized assuming a set of vertical wells placed at the best $\mathrm{PP}_{\mathrm{C}} \cap \mathrm{PP}_{\mathrm{L}}$, supported by the additional considerations of minimizing the water saturation and respecting a minimum distance between wells. The results, compared to the production of the original grouped UNISIM-I-D and grouped PUNQ-S3 wells, show a reasonable performance of the PP strategy for well placement. In addition, the net present value shows that the proposed wells are economically feasible. The study provided that under certain conditions, $\mathrm{PP}_{\mathrm{C}} \cap \mathrm{PP}_{\mathrm{L}}$ is an alternative approach to find out target positions exhibiting a high productivity potential for well placement appropriateness and oil recovery.
\end{abstract}

Keywords Productivity potential $\cdot$ Proxy function $\cdot$ Production simulations $\cdot$ UNISIM-I-D synthetic reservoir $\cdot$ PUNQ-S3 synthetic reservoir

\section{Introduction}

Porosity and permeability are two important reservoir parameters. The first is related to the stock capacity of fluids in the rock while the second is responsible for the mobility of the fluids inside the pore networks of the rock. Before any action can be taken, for oil recovery from a reservoir, quite a lot of problems are involved specifically related to find out strategies for well placement. It is known that the ultimate purpose of an oil field development is getting an efficient extraction of hydrocarbons from the reservoir. In this regard, the relevance of well placement and perforations

Waldir L. Roque

roque@ci.ufpb.br

1 Laboratório de Modelagem em Engenharia de Petróleo - LaMEP, Universidade Federal da Paraíba - UFPB, João Pessoa, PB, Brazil strategies for recovery efficiency have to be carefully taken into account. Several investigations and analyses have to be done before proposing a strategy to foresee a reservoir target area that provides an indication of high potential for oil recovery. In other words, some feasible positions presenting the best productivity potential (PP) according to some criteria are necessary.

A number of approaches have been presented and discussed in the literature for at least fifteen years. In Kharghoria et al. (2003), a heuristic model for selection of reservoir drilling targets based on a productivity proxy function was proposed. This proxy function considered a combination of petrophysical, geometrical and dynamical attributes of each grid block, and they have shown that this function had a good correlation to the production potential of wells intersecting these grid blocks. In Guerra and Narayanasamy (2005), a productivity potential map (PPM) was proposed as an approach to delineate reservoir regions with favorable production potential for well placement considering a proxy 
function as a combination of petrophysical properties resembling Darcy's law. The results have shown that well locations selected using PPM technique presented better flow performance when compared to the conventional stock tank oil initially in place (STOIIP)-based approach. Later on, Min et al. (2011) used a productivity potential proxy function as input data to feed an artificial neural network to predict optimal drilling location that maximizes the cumulative oil production without reservoir simulations. The productivity potential used by them has shown to be more useful than the permeability to predict the cumulative production, as it included additional valuable information like flow and storage capability to an artificial neural network.

Liu and Jalali (2006) have proposed a dynamic productivity potential proxy function that took into account a combination of three terms: absolute permeability, oil saturation and oil phase pressure at the grid block, and additionally, the distance of well location to the nearest reservoir boundary was considered. Later on, Ding et al. (2014) proposed and extension of Liu and Jalali's proxy model taking into account the negative effect of bottom water and gas cap on field development. Their method combines a modified particle swarm optimization algorithm (MPSO) and quality map (QM) with the goal to optimize well placement. More recently, Ding et al. (2019) introduced the direct mapping of productivity potential (DMPP) as a QM to represent regions with high- and low-productivity potential in the reservoir that is used to determine the threshold value of productivity potential based on their time-dependent proxy function. This method was applied to the PUNQ-S3 synthetic reservoir model showing that well locations based on DMPP had a better flow performance compared to the conventional oil reserves abundance map (ORAM) approach.

Even with these new approaches to well placement considering some proxy functions to identify productivity potential regions, a simple criterium that has been proposed in the literature is the use of a productivity proxy function based on the oil-bearing capacity of each grid block, represented by the product of porosity and oil saturation, $\phi S_{\mathrm{o}}$, and, on the other hand, by the mobility capacity of oil that may be represented by the product of absolute permeability and oil saturation, $k S_{\mathrm{o}}$. However, so far, this approach has yet not been looked over under the light of production simulations.

In this paper, we present and discuss a strategy and the appropriateness of a PP proxy function to well placement in a reservoir based on a set of production simulations. To carry on this investigation, it will be considered the UNISIM-I-D synthetic reservoir model (Avansi and Schiozer 2015) that was developed taking into account actual data from Namorado field, located in Campos basin, Brazil, and also, the PUNQ-S3 synthetic reservoir model (Floris et al. 2001) that was developed by a group of companies, institutes and universities in the European Union based on data of a real field located in the North Sea. The simulations will be done with $\mathrm{CMG}^{1}$ software suite using the black oil model.

\section{Development}

A simple productivity potential (PP) proxy function has been proposed (Kharghoria et al. 2003; Guerra and Narayanasamy 2005; Min et al. 2011) as,

$\mathrm{PP}_{i}=\phi_{i} k_{i} S_{\mathrm{o}, i}^{n}$,

where $\phi_{i}, k_{i}$ and $S_{\mathrm{o}, i}$ are the porosity, absolute permeability and oil saturation at the $i$ th active grid block, respectively, and $n$ is a correlation constant. In Min et al. (2011), the study was conducted with $n=1$, as will be considered in the current study too. Notice that, $\mathrm{PP}_{i}$ has the same dimension of permeability.

\section{PP strategic equations}

To be able to discuss the well placement and perforation strategies, let define the unit-based normalization of the productivity potential $\mathrm{PP}_{\mathrm{N}}$, the column-wise $\mathrm{PP}_{\mathrm{C}}$ value and the layer-wise $\mathrm{PP}_{\mathrm{L}}$ value over the reservoir grid blocks, by

$\mathrm{PP}_{\mathrm{N}, i}=\frac{\mathrm{PP}_{i}-\mathrm{PP}_{\min }}{\mathrm{PP}_{\max }-\mathrm{PP}_{\min }}$,

where $\mathrm{PP}_{\max }$ and $\mathrm{PP}_{\min }$ are the maximum and minimum values of $\mathrm{PP}_{i}$, respectively, leading to $0 \leq \mathrm{PP}_{\mathrm{N}, i} \leq 1$;

$\mathrm{PP}_{\mathrm{C}, j}=\frac{1}{T} \sum_{i=1}^{T} \mathrm{PP}_{\mathrm{N}, i}$,

where $j$ runs over all reservoir columns and $T$ is the number of blocks at column $j$;

$\mathrm{PP}_{\mathrm{L}, j}=\frac{1}{M} \sum_{i=1}^{M} \mathrm{PP}_{\mathrm{N}, i}$,

where $j$ runs over all reservoir layers and $M$ is the number of blocks at layer $j$.

As a strategy for well placement based on the PP proxy equations, a couple of production simulations will be realized assuming a vertical well placed at the highest $\mathrm{PP}_{\mathrm{C}}$ and perforations done hierarchically at grid blocks with the highest values of $\mathrm{PP}_{\mathrm{L}} \cdot \mathrm{PP}_{\mathrm{C}}$ and $\mathrm{PP}_{\mathrm{L}}$ are column-wise and layer-wise productivity potentials. To verify this hypothesis,

\footnotetext{
${ }_{1}^{1}$ https://www.cmgl.ca/.
} 


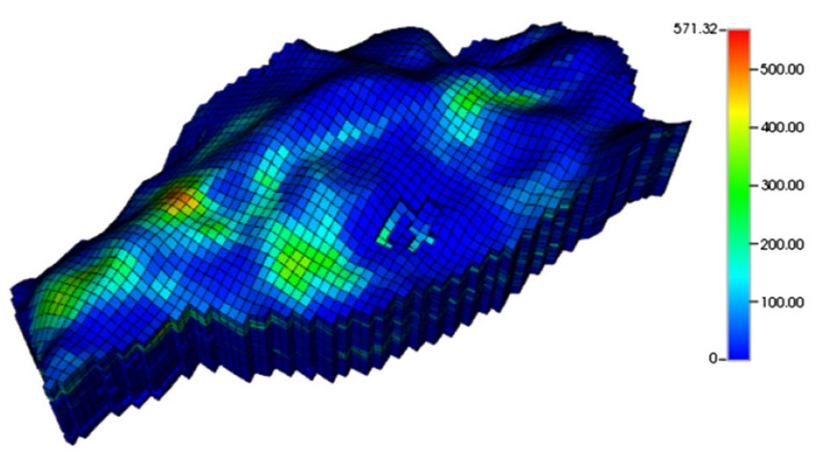

Fig. $1 \mathrm{PP}_{i}$ map for the active blocks of the reservoir

the UNISIM-I-D and PUNQ-S3 synthetic reservoirs will be considered.

\section{Reservoir synthetic models}

To carry our production simulations based on the PP approach, we will consider the following synthetic reservoirs: (1) UNISIM-I-D is a synthetic reservoir with grid dimensions $i=81, j=58, l=20$, where $i, j$ are horizontal and $l$ in-depth coordinates, with 36, 403 active blocks and block volume of $100 \times 100 \times 8 \mathrm{~m}^{3}$ upscaled from its high resolution grid. Complete details about UNISIM can be found in Avansi and Schiozer (2015) and https://www.unisi m.cepetro.unicamp.br/. (2) PUNQ-S3 is a synthetic reservoir with a grid dimension of $i=19, j=28, l=5$, with 1761 active blocks and a block volume of $180 \times 180 \times 5 \mathrm{~m}^{3}$, additional details can be found in Floris et al. (2001) and https:// www.imperial.ac.uk/earth-science/research/researchgroups/ perm/.

To compute Eqs. 1-4, we are considering the UNISIMI-D and PUNQ-S3 data files as provided by the synthetic reservoir developers and used the CMG software suite to run the simulations. In Eq. 1, the porosity $\phi_{i}$ is considered as the effective porosity, that is, the porosity corrected by the net-to-gross (NTG) ratio, and $k_{i}$ is the norm of the three permeability directional values at the grid block.

\section{UNISIM-I-D}

In what follows, the results of Eqs. 1-4 are displayed as $3 D$ reservoir maps. Figure 1 shows the $\mathrm{PP}_{i}$ reservoir map of the active blocks.

Figure 2 shows the $\mathrm{PP}_{\mathrm{N}}$ reservoir map (left) and the $\mathrm{PP}_{\mathrm{N}}$ histogram (right) for the active blocks. Notice that, nearly
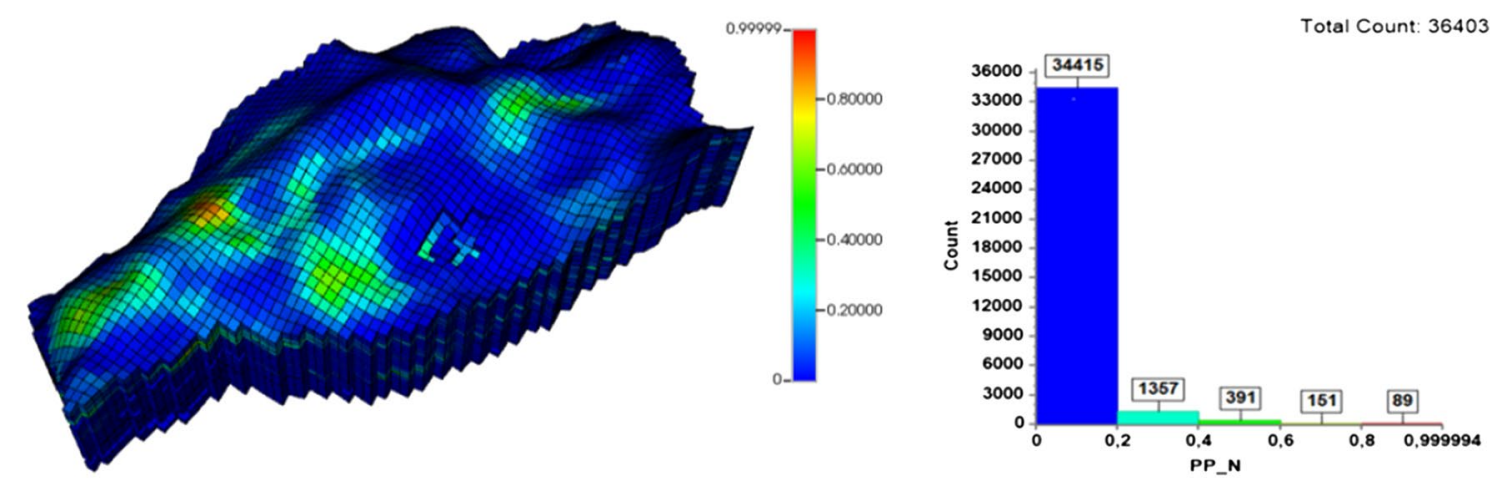

Fig. 2 Normalized $\mathrm{PP}_{i}$ map (left) and its histogram for the active blocks
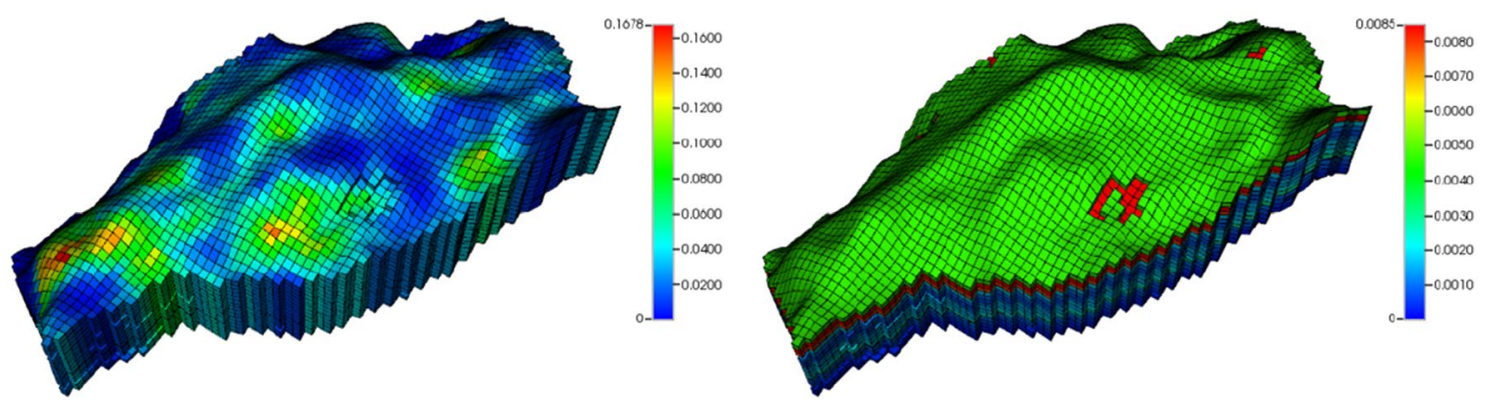

Fig. $3 \mathrm{PP}_{\mathrm{C}}$ (left) and $\mathrm{PP}_{\mathrm{L}}$ (right) maps 


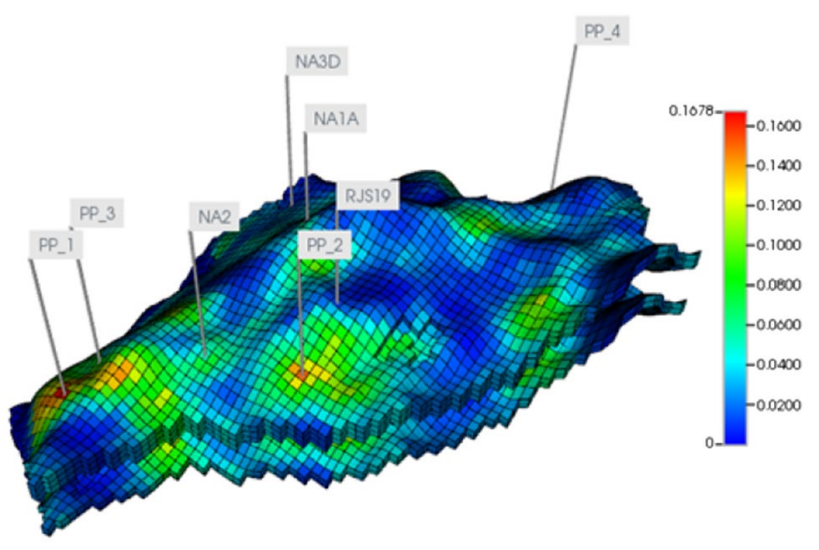

Fig. 4 Illustration of PP_1 to PP_4 wells placed at best $\mathrm{PP}_{\mathrm{C}}$ and the best five $\mathrm{PP}_{\mathrm{L}}$

$95 \%$ of the active blocks have a very low $\left(0 \leq \mathrm{PP}_{\mathrm{N}} \leq 0.2\right)$ normalized productivity potential.

Figure 3 shows the $P P$ column $\left(\mathrm{PP}_{\mathrm{C}}\right)$ and the $P P$ layer $\left(\mathrm{PP}_{\mathrm{L}}\right)$ maps of the reservoir.

The best reservoir $\mathrm{PP}_{\mathrm{C}}$ is located at active block $[i, j, l]=[10,45,1]$. According to the hypothesis, a vertical well, say PP_1, will be placed at this block, and perforations along the column will be set in a sequence according to the intersection of the column with hierarchically decreasing values of $\mathrm{PP}_{\mathrm{L}}$. In addition, the perforations will be set on those active blocks with the minimum water saturation value, $S_{w}=0.17$, of the reservoir. Here, we have also considered a minimum Euclidean distance limit of at least $500 \mathrm{~m}$ between the wells and avoid the border grid blocks.
Figure 4 gives an illustration of the four original wells NA1A, NA2, NA3D, RJS19 provided by UNISIM-I-D and the best four proposed PP_1, PP_2, PP_3 and PP_4 wells placed according to the PP strategy.

Table 1 gives for each well its perforation blocks, its $\mathrm{PP}_{\mathrm{C}}$ and $\mathrm{PP}_{\mathrm{L}}$ values. Notice that, the total number of perforations of the PP wells is 38 , while the UNISIM-I-D well perforation number is larger, 47 , and that $\mathrm{PP}_{\mathrm{C}}$ values of UNISIM-I$\mathrm{D}$ wells are very low, although the well placement criterium for them was not based on the current approach.

\section{PUNQ-S3}

For the PUNQ-S3 reservoir, Fig. 5 shows the $\mathrm{PP}_{\mathrm{N}}$ reservoir map and the $\mathrm{PP}_{\mathrm{N}}$ histogram for the active blocks. Notice that, $76 \%$ of the active blocks have a very low normalized productivity potential $\left(0 \leq \mathrm{PP}_{\mathrm{N}} \leq 0.2\right)$.

Figure 6 shows the PP columns and the PP layers' maps of the reservoir. The best reservoir $\mathrm{PP}$ column $\left(\mathrm{PP}_{\mathrm{C}}\right)$ is located at active block $[i, j, l]=[15,24,1]$. According to the hypothesis, a vertical well, say PP_NTG_1, will be placed at this block and perforations will be set in a sequence according to the intersection of the column with the hierarchically decreasing values of $\mathrm{PP}$ layers $\left(\mathrm{PP}_{\mathrm{L}}\right)$. In addition, the perforations will be done on those active blocks with minimum water saturation value $S_{w}=0.20$.

Figure 7 gives an illustration of the six original wells PRO-1, PRO-4, PRO-5, PRO-11, PRO-12 and PRO-15 provided by PUNQ-S3 and the best six proposed wells PP NTG_1 to PP_NTG_6 placed at $\mathrm{PP}_{\mathrm{C}} \cap \mathrm{PP}_{\mathrm{L}}$. Here, well's placement is considered within a minimum Euclidean
Table 1 UNISIM-I-D and PP wells with their respective perforation blocks, $\mathrm{PP}_{\mathrm{C}}$ and $\mathrm{PP}_{\mathrm{L}}$

\begin{tabular}{llllllllll}
\hline Wells & NA1A & NA2 & NA3D & RJS19 & PP_1 & PP_2 & PP_3 & PP_4 & PP $_{L}$ \\
\hline Perf blocks & 38361 & 21361 & 44431 & 31271 & 10451 & 24261 & 14411 & 66221 & 0.0364 \\
& 38262 & 21362 & 44432 & 31272 & 10452 & 24262 & 14412 & 66221 & 0.0659 \\
& 38363 & 21363 & 44433 & 31273 & 10453 & 24263 & 14413 & 66223 & 0.0609 \\
& 38366 & 21366 & 44436 & 31276 & 10456 & 24266 & 14416 & 66226 & 0.0140 \\
& 38367 & 21367 & 44437 & 31277 & 10457 & 24267 & 14417 & 66227 & 0.0083 \\
& 38368 & 21368 & 44438 & 31278 & 10458 & 24268 & 14418 & 66228 & 0.0059 \\
& 38369 & 21369 & - & 31279 & - & 24269 & 14419 & 66229 & 0.0087 \\
& 383610 & 213610 & 444310 & 312710 & - & 242610 & 144110 & 662210 & 0.0121 \\
& 383611 & 213611 & 444311 & 312711 & - & 242611 & 144111 & 662211 & 0.0127 \\
& 383612 & 213612 & - & 312712 & - & 242612 & - & 662212 & 0.0159 \\
& 383613 & - & - & 312713 & - & - & - & 662213 & 0.0219 \\
& 383615 & - & - & 312715 & - & - & - & 662215 & 0.0080 \\
& 383616 & - & - & 312716 & - & - & - & 662216 & 0.0062 \\
& 383617 & - & - & - & - & - & - & - & 0.0039 \\
PP $_{C}$ & 383618 & - & - & - & - & - & - & - & 0.0270 \\
\hline & 383619 & - & - & - & - & - & - & - & 0.0029 \\
& 0.0333 & 0.0532 & 0.0129 & 0.0118 & 0.1678 & 0.1494 & 0.1415 & 0.1381 & \\
\hline
\end{tabular}



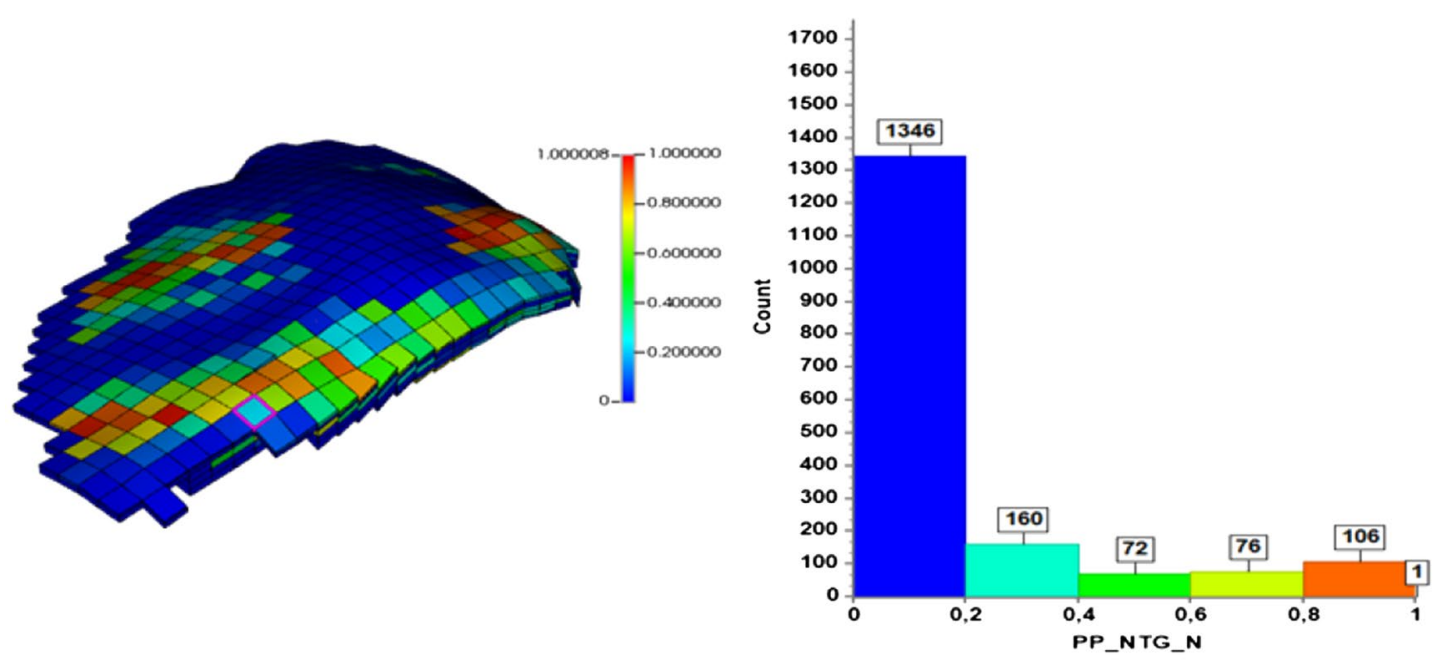

Fig. 5 Normalized $\mathrm{PP}_{i}$ map (left) and its histogram for the active blocks
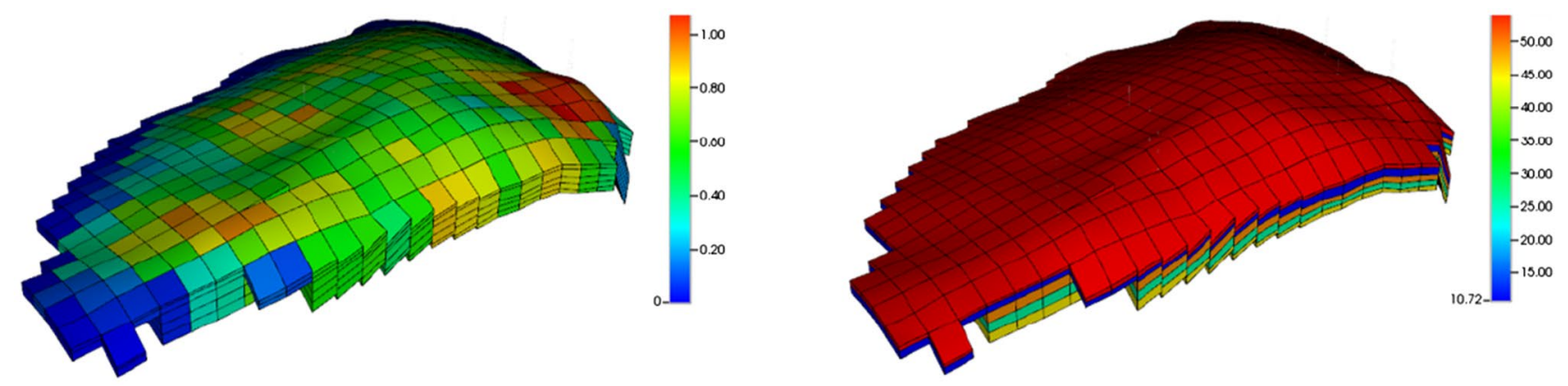

Fig. $6 \mathrm{PP}_{\mathrm{C}}$ (left) and $\mathrm{PP}_{\mathrm{L}}$ (right) maps

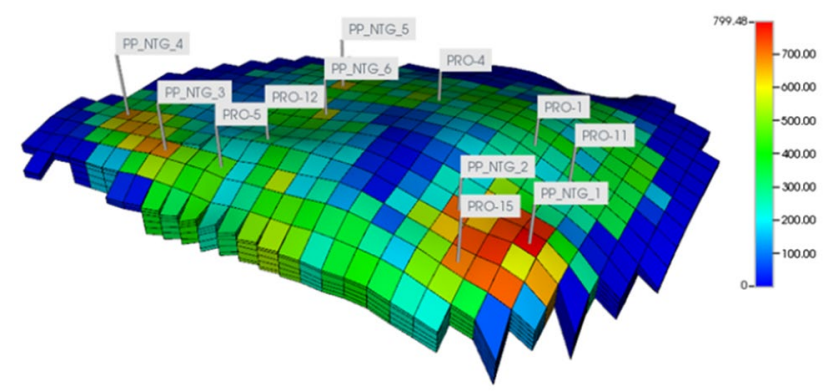

Fig. 7 PP_NTG_1 to PP_NTG_6 placed at best $\mathrm{PP}_{\mathrm{C}}$ and $\mathrm{PP}_{\mathrm{L}}$

distance limit of at least $430 \mathrm{~m}$ from each other and avoiding border grid blocks, similarly to what is presented by the original PRO wells.

Table 2 gives the perforation blocks, the $\mathrm{PP}_{\mathrm{C}}$ and $\mathrm{PP}_{\mathrm{L}}$ values for PUNQ-S3 PRO wells and PP_NTG wells. Notice that, in Table 2, the $\mathrm{PP}_{\mathrm{C}}$ value for the PRO-15 is higher than the value for the PP_NTG_(4,5,6). In fact, PP_NTG_4 could not be placed at $\mathrm{PP}_{\mathrm{C}}=0.3921$ column because it is neighbor to PP_NTG_2, corresponding to a distance shorter than $430 \mathrm{~m}$, which is to be avoided.

\section{Production simulations}

The purpose of production simulations is to verify the behavior of the proposed wells in terms of oil recover productivity and economical viability in comparison with the production of the original UNISIM-I-D and PUNQ-S3 wells.

\section{UNISIM-I-D case}

Considering first the UNISIM-I-D reservoir, which presented the total oil in place of $0.13029 \times 10^{9} \mathrm{~m}^{3}$, total water in place of $0.12249 \times 10^{9} \mathrm{~m}^{3}$ and the total gas in place of $0.14781 \times 10^{11} \mathrm{~m}^{3}$; the vertical well PP_1 is perforated 


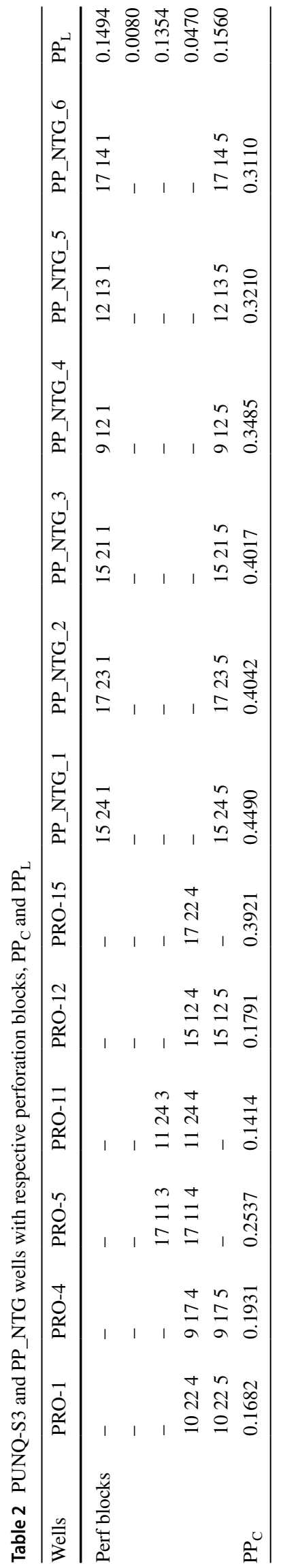

in 6 active blocks, PP $\_2$ in 10 active blocks, PP $\_3$ in 9 active blocks and PP_4 in 13 active blocks, according to the methodology and observing the condition of minimum water saturation value $S_{w}=0.17$.

A series of simulations were run to see the production behavior of the proposed wells versus the historical production behavior of the four original wells, provided by UNISIM-I-D synthetic reservoir. All simulations were done using CMG software suite considering the black oil model.

Figure 8 shows the results of cumulative oil (COP-SC) and cumulative liquid (CLP-SC) productions, over a time span of 20 years, for the original wells and the proposed ones. It can be observed that PP_2 and PP_4 COP-SC productions are higher than the individual production of anyone of the original wells. A remark here is that PP_2 and PP_4 have more perforations than PP_1 and PP_3. Notice that, the NA1A well is the third best COP-SC producer among all wells and the best among the original UNISIM-I-D wells. The CLP-SC, which is the oil plus water production, indicates that the proposed wells PP_2 and PP_4 perform better.

Figure 9 shows the cumulative water (CWP-SC) and the water oil ratio (WOR-SC) of the wells. It can be seen that both PP_1 and PP_4 have higher CWP-SC than the other wells during most of the simulation time span.

The WOR-SC simulation shows that the PP_1 well has a high water oil ratio pick reaching approximately 4.0 and then slows down slowly. On the other hand, all the other wells present a lower water oil ratio along the production time span. Therefore, it becomes important to compare the behavior of the overall production of the original wells together versus the proposed wells together.

Let group the two sets of wells, one formed by the UNISIM-I-D wells (NA1A, NA2, NA3D and RJS19) and the other formed by the proposed ones (PP_1, PP_2, PP_3 and PP_4). Figure 10 shows the cumulative oil and the cumulate water productions for the U-Wells (UNISIM-Wells, dashed line) and PP-Wells (proposed wells, solid line).

From the simulation results shown in Fig. 10, the grouped PP-Wells has a higher cumulative oil and cumulative water productions than the grouped U-Wells. Figure 11 shows the oil rate and the water rate, both yearly, for the U-Wells and the PP-Wells. In the oil rate simulation, it can be seen that the PP-Wells production rate is higher than the U-Wells along the time span. Besides that, the water rate of the PP-Wells is higher than that of the U-Wells. Therefore, under these circumstances an immediate question arises: which one of the grouped wells would reveal as better, economically speaking.

The net present value (NPV) is an important economic measure for projects or equipments that takes into account discount factors and cash flow and has been of much use by the oil industry Fanchi (2010) and CMOST AI 
Fig. 8 COP-SC (a) and CLP-SC (b) for UNISIM and PP wells

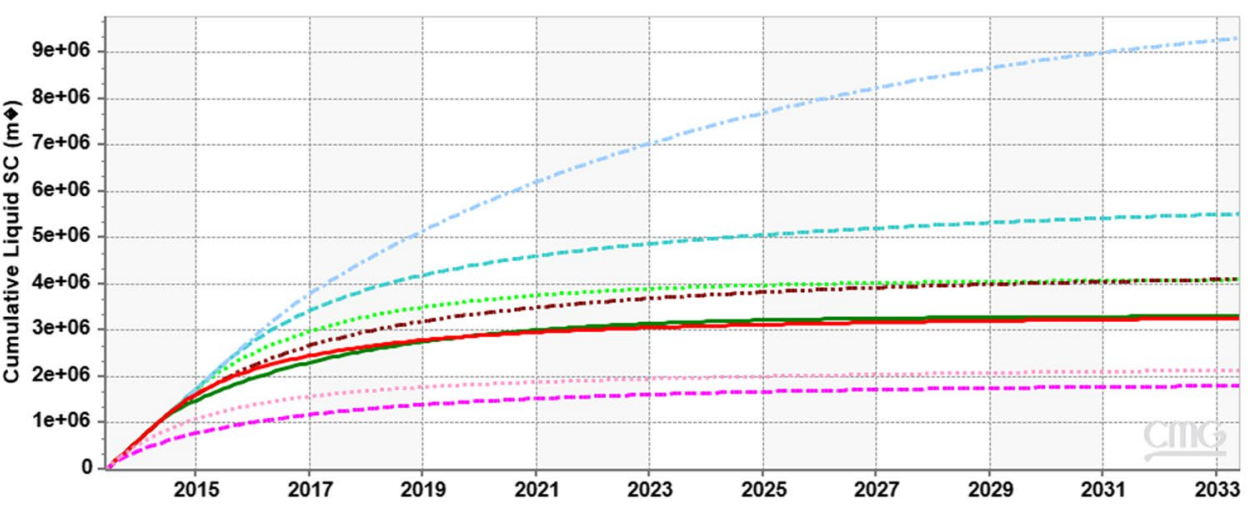

— PP_1, Cumulative Liquid SC, WLR1_PP_UNISIM-I-D.sr3 -- PP_2, Cumulative Liquid SC, WLR1_PP_UNISIM-I-D.sr3 .... PP_3, Cumulative Liquid SC, WLR1_PP_UNISIM-I-D.sr3 $\ldots$ PP_4, Cumulative Liquid SC, WLR1_PP_UNISIM-I-D.sr3 -.. NA1A, Cumulative Liquid SC, WLR1_PP_UNISIM-I-D.sr3 — NA2, Cumulative Liquid SC, WLR1_PP_UNISIM-I-D.sr3 -- NA3D, Cumulative Liquid SC, WLR1_PP_UNISIM-I-D.sr3 … RJS19, Cumulative Liquid SC, WLR1_PP_UNISIM-I-D.sr3

(a)

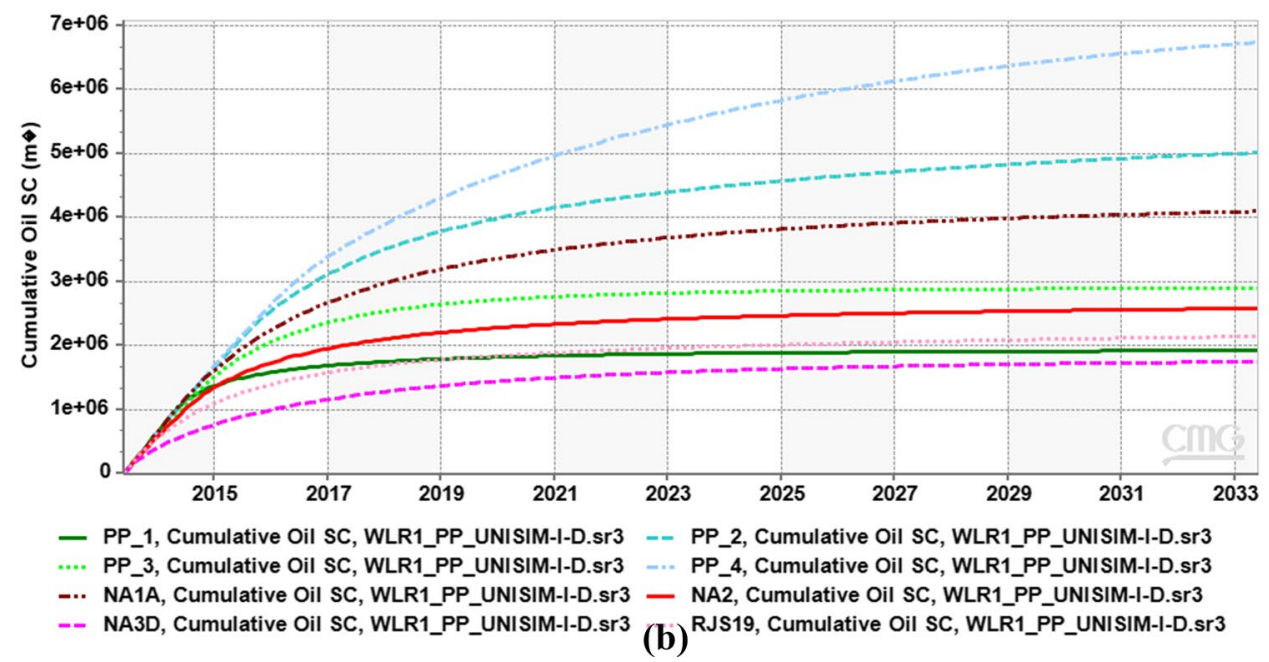

UserManual (2019). To see if the PP-Wells are economically profitable compared to the U-Wells, even presenting a higher water rate than U-Wells, the NPV was estimated. To do the NPV computations, the oil price was assumed as $60 \$ / \mathrm{bbl}$ and the water treatment as $1 \$ / \mathrm{bbl}$. However, the actual costs are not so relevant as the calculations are done for both PP-Wells and U-Wells using the same values.

Considering the oil rate yearly and water rate yearly data, as presented in Fig. 11, the NPV for both groups were found to be positive, which indicates that they are viable investments. However, U-Wells presented a smaller NPV than PP-Wells, whose profit would correspond to $26 \%$ of the PP-Wells. Therefore, the additional completion of the set of proposed PP-Wells would be a worth investment as it overcomes the UNISIM-I-D original wells' profit.

On the other hand, taking into account an initial completion investment, if it is considered either the isolated U-Wells or the PP-Wells production simulations, the NPV estimated for each group individually has shown that the
PP-Wells completions would be a better investment, with a profit margin of $23 \%$ over the U-Wells.

From these results, it can be seen that the methodology, at least in this case study, seems an interesting strategy for well placement and oil recovery.

\section{PUNQ-S3 case}

Now let us consider the PUNQ-S3 reservoir, which presented the total oil in place of $0.17373 \times 10^{8} \mathrm{~m}^{3}$, total water in place of $0.12568 \times 10^{8} \mathrm{~m}^{3}$ and the total gas in place of $0.16503 \times 10^{10} \mathrm{~m}^{3}$. The six PRO wells in the PUNQ-S3 synthetic reservoir had a series of events specified by constrains (targets) along the time span; however, to simplify the production simulations, without loss of generality, these events were discarded. This allowed an uniformity between them and the PP_NTG-Wells.

As the production simulations of the PUNQ-S3 reservoir wells follow a similar behavior as the UNISIM-I-D 
Fig. 9 CWP-SC (a) and WORSC (b) for UNISIM and PP wells

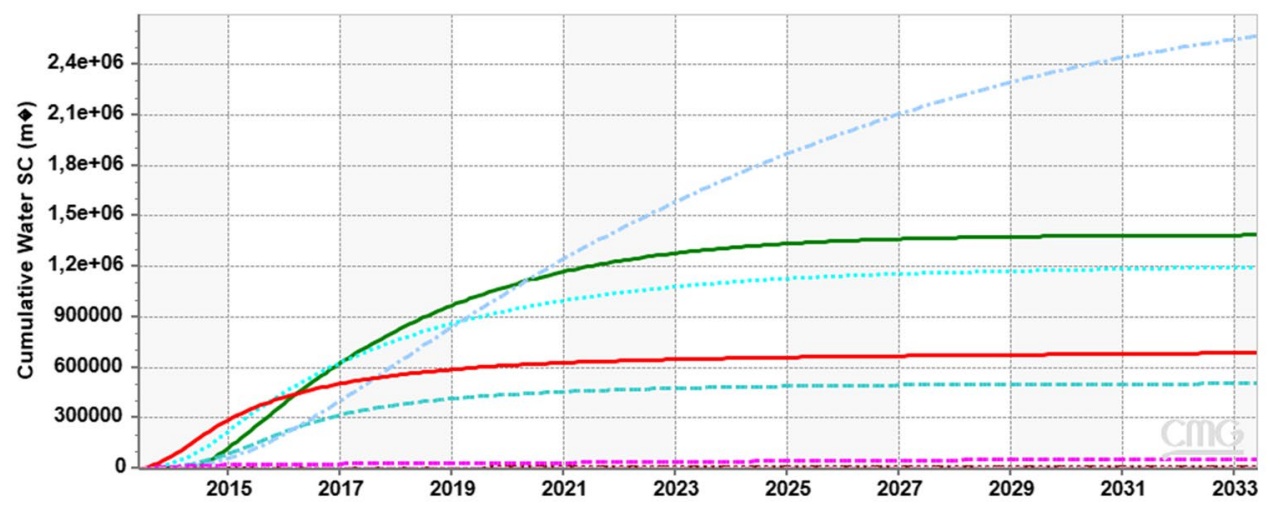

- PP_1, Cumulative Water SC, WLR1_PP_UNISIM-I-D.sr3 -- PP_2, Cumulative Water SC, WLR1_PP_UNISIM-I-D.sr3 .... PP_3, Cumulative Water SC, WLR1_PP_UNISIM-I-D.sr3 -.. PP_4, Cumulative Water SC, WLR1_PP_UNISIM-I-D.sr3 -.. NA1A, Cumulative Water SC, WLR1 PP UNISIM-I-D.sr3 - NA2, Cumulative Water SC, WLR1 PP UNISIM-I-D.sr3 -- NA3D, Cumulative Water SC, WLR1_PP_UNISIM-I-D.sr3 … RJS19, Cumulative Water SC, WLR1_PP_UNISIM-I-D.sr3

(a)

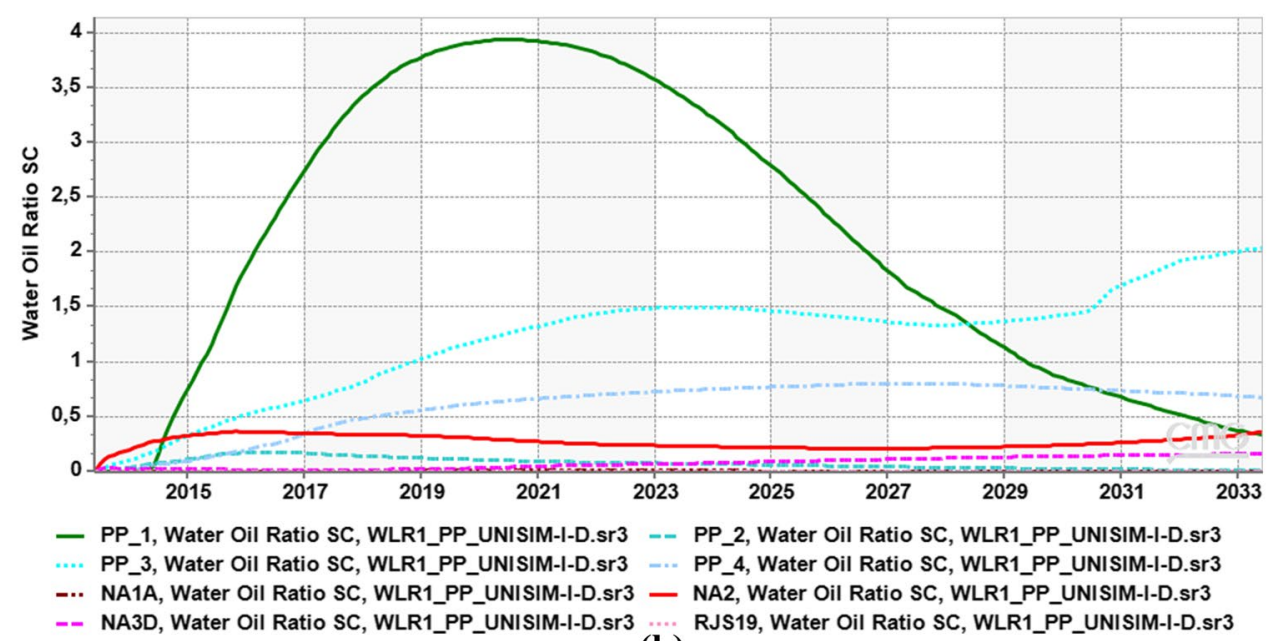

(b) reservoir, only the most relevant aspects will be presented. Let assume that the groups of PRO-Wells and PP_NTGWells are independently placed in the reservoir. Figure 12 shows the yearly oil rate (OR), water rate (WR) and gas rate (GR) simulations for these groups.

From the simulations shown in Fig. 12, it can be observed that the proposed PP_NTG-Wells present a slightly lower oil rate, a similar gas rate and slightly higher water rate than the PRO-Wells. These results indicate that the PRO-Wells had a slight better production performance compared to the PP_NTG-Wells, even having one less perforation and their $\mathrm{PP}_{\mathrm{C}} \cap \mathrm{PP}_{\mathrm{L}}$ being inferior, as pointed out in Table 1 .

To see the economic viability of the proposed wells related to the oil versus water production, the net present value (NPV) was estimated. The NPV for both groups were found to be positive, but the profit of the PP_NTG-Wells grouped corresponds to $83 \%$ of the PRO-Wells, which indicates that despite both to be profitable, the proposed PP_ NTG wells would provide a less return on investment.

\section{Discussion}

The methodology proposed here, based on a simple productivity potential proxy function (see Eq. 1), has been used to investigate its appropriateness as a strategy for well placement. According to the methodology, well placements were proposed to be set at the intersection of the best productivity potential column $\mathrm{PP}_{\mathrm{C}}$ and perforated at the best productivity potential layers $\mathrm{PP}_{\mathrm{L}}$, and assuming a least distance between the wells, that perforations would be done at active blocks with the minimum water saturation and avoiding reservoir borders.

To investigate the feasibility of the proposed approach, the UNISIM-I-D synthetic reservoir, derived from Namorado field data, Campos basin, Brazil, and the PUNQ-S3 synthetic reservoir, derived from a North See oil field, were used for a set of production simulations; in particular, the yearly oil and water rate at standard conditions were realized to compare the sets of grouped wells, i.e., the 
Fig. 10 COP-SC (a) and CWP$\mathrm{SC}(\mathbf{b})$ for grouped U-Wells and PP-Wells

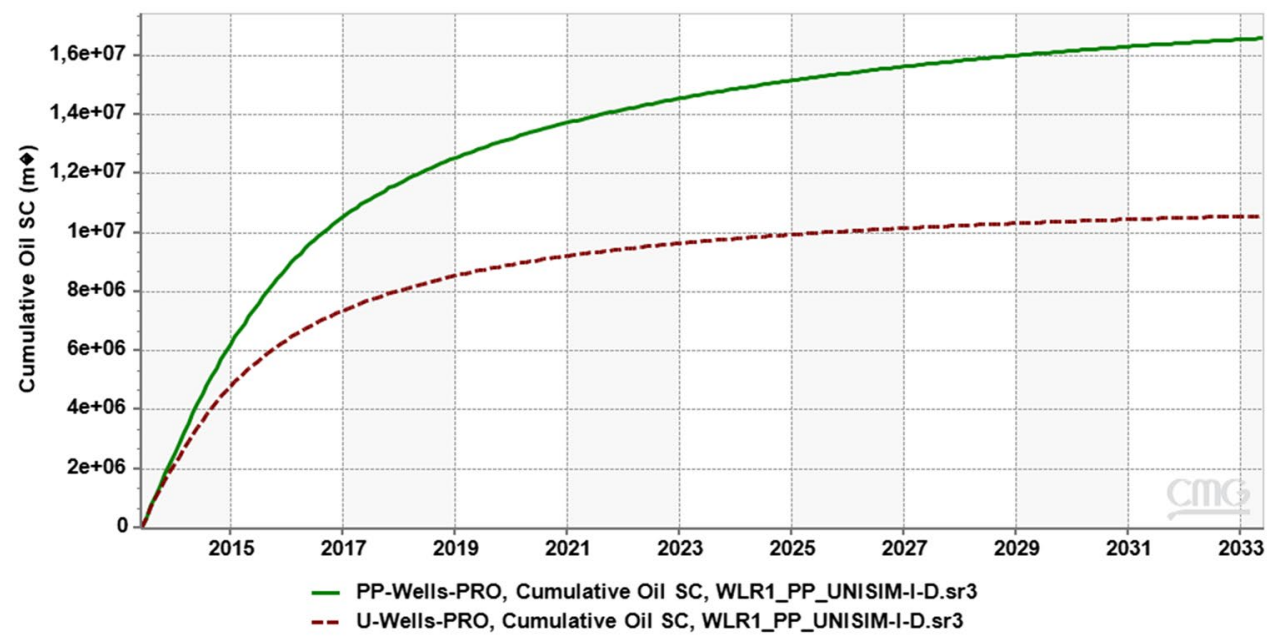

(a)

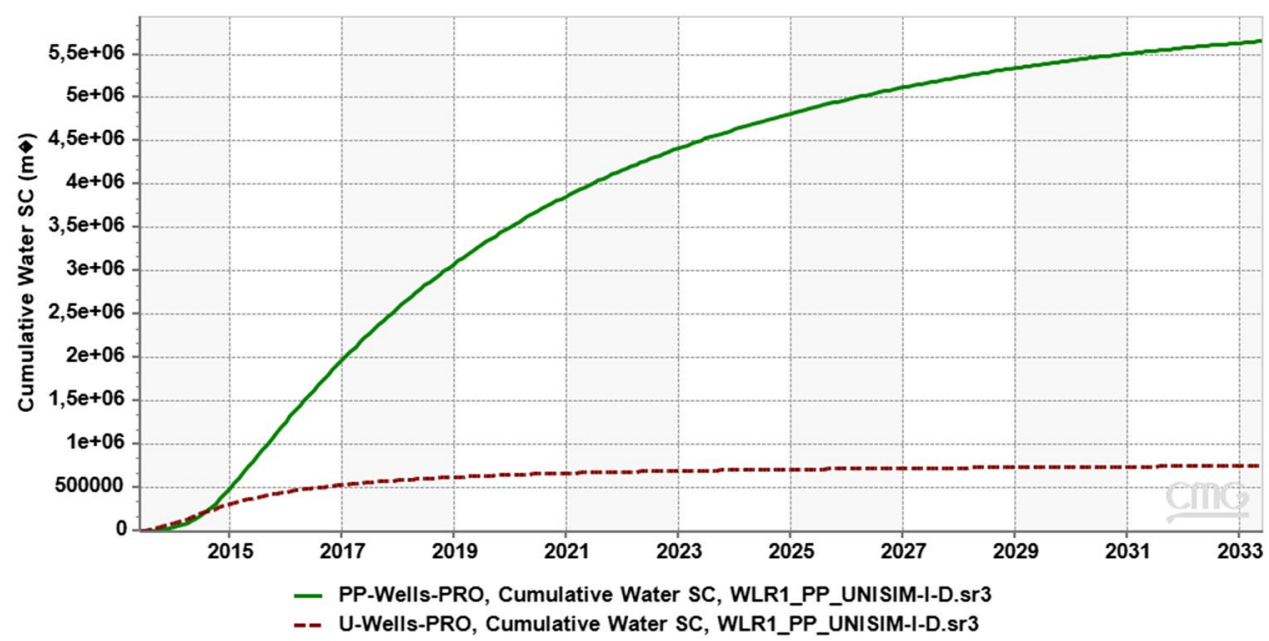

(b) original UNISIM-I-D wells (U-Wells) versus the proposed wells (PP-Wells), and the original PUNQ-S3 wells (PROWells) versus the proposed wells (PP_NTG-Wells).

The simulation results have shown that the oil rate of the PP-Wells overcomes the oil production rate of the U-Wells during the same simulation time span (20 years). But, at the same time, simulations have shown that the water rate of the PP-Wells is higher than that of the U-Wells, too (see Fig. 11). However, according to the net present value (NPV) estimated for both groups, the U-Wells' profit would be only $26 \%$ of the PP-Wells. On the other hand, when the U-Wells or PP-Wells were placed as an isolate group on the reservoir, the PP-Wells' NPV exhibits a profit of $23 \%$ over the U-Wells.

When individual wells are observed, the cumulative oil production, whose graph is given in Fig. 8, shows that the performance of the PP_1 well is inferior than those of PP_2, PP_3 and PP_4, and PP_3 is inferior to PP_2. Yet from
Fig. 8, the NA2 COP is higher than the PP_1, which looks like an inconsistency. From Table 1, it can be seen that the NA2 well has a lower $\mathrm{PP}_{\mathrm{C}}$ value compared to PP_1, but 4 more perforations than PP_1, in blocks with water saturations up to $S_{w}=0.2$. Similarly, NA1A COP performance is better than PP_1 and also PP_3 as it has more perforations blocks with $S_{w}=0.17$ than PP_1 and PP_3. Therefore, it can be seen that although NA1A and NA2 wells have a lower $\mathrm{PP}_{\mathrm{C}} \cap \mathrm{PP}_{\mathrm{L}}$ values, the number of perforations in low water (high oil) saturations may overcomes the oil recovery performance compared to wells with better $\mathrm{PP}_{\mathrm{C}} \cap \mathrm{PP}_{\mathrm{L}}$ values, but a lower number of perforations.

In the case study of the PP_NTG-wells versus the PROWells, the production simulations have shown that the proposed wells presented a slightly lower oil production rate than the original PUNQ-S3 PRO-Wells. The NPV analysis done to compare both group of wells shows that the profit in 
Fig. 11 OR-SC (a) and WR-SC (b) for grouped U-Wells and PP-Wells

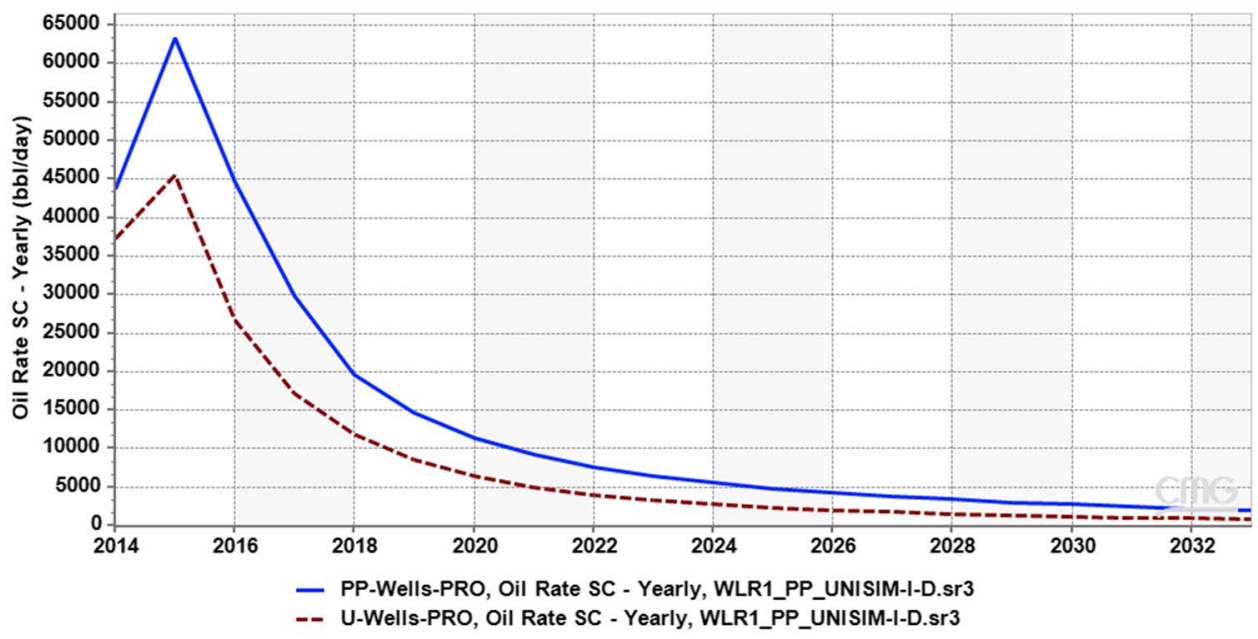

(a)

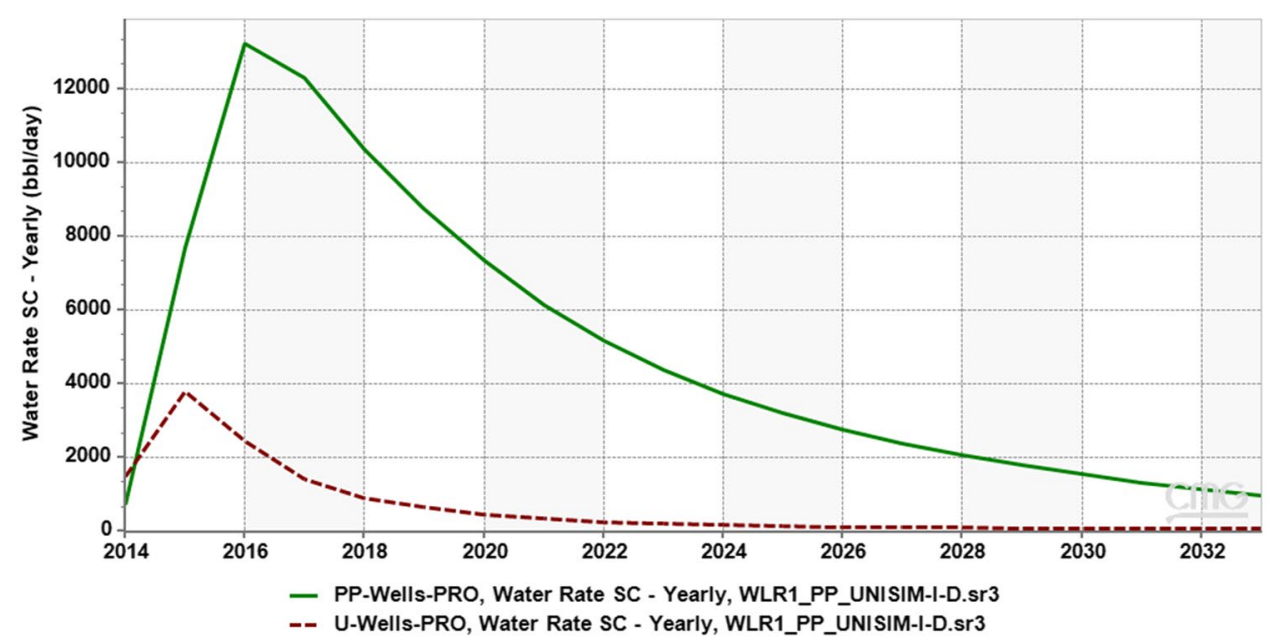

(b) oil production of the proposed PP_NTG-Wells would represent only $83 \%$ of the expected profit for the PRO-Wells.

When production simulations are observed for PRO wells individually and for PP_NTG wells individually, a similar behavior that have occurred to individual U-Wells and PPWells happens. This means that the hierarchical sequence of $\mathrm{PP}_{\mathrm{C}} \cap \mathrm{PP}_{\mathrm{L}}$ is not sufficient to isolatedly guarantee the hierarchy of well placement in terms of better oil production. Although this shows a weakness of the PP strategy, however, when the number of wells to be complete is known a priori, the current PP strategy might be useful compared to and competitive with other well placement techniques based on oil recovery performance from production simulations.

It is worthwhile to mention here that once Eqs. 1-4 are introduced into the CMG simulator, the computations to identify the positions for well placement, according to the PP strategy, is quite simple, although this requests some user manual intervention. After that, the production simulations are automatic and run in less than a few minutes according to the number of active grid blocks in the reservoir and the production time span required.

For both synthetic reservoir studied here, just the simulations' run were less than $3 \mathrm{~min}$. Therefore, the whole process of PP strategy is not time-consuming, which allows experimenting alternative set of positions for well placement and production simulations. In fact, a couple of alternative wells were placed in both UNISIM-I-D and PUNQ-S3 reservoir following the PP strategy, but setting them at positions outside the main hierarchical sequence (first four PP-Wells and first six PP_NTG-Wells). However, the oil production simulations for grouped wells have shown always to be smaller than that indicated by the main hierarchical sequence provided by the PP strategy. 
Fig. 12 Oil, water and gas rates for PRO-Wells (a) and PP_NTG-Wells (b)

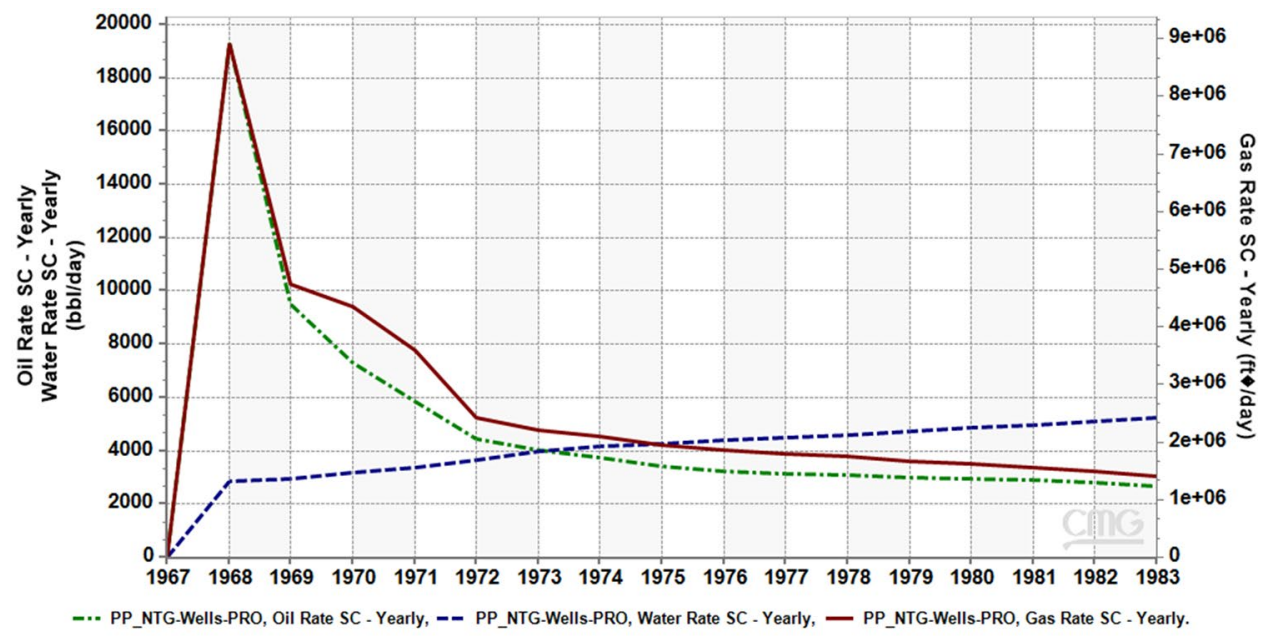

(a)

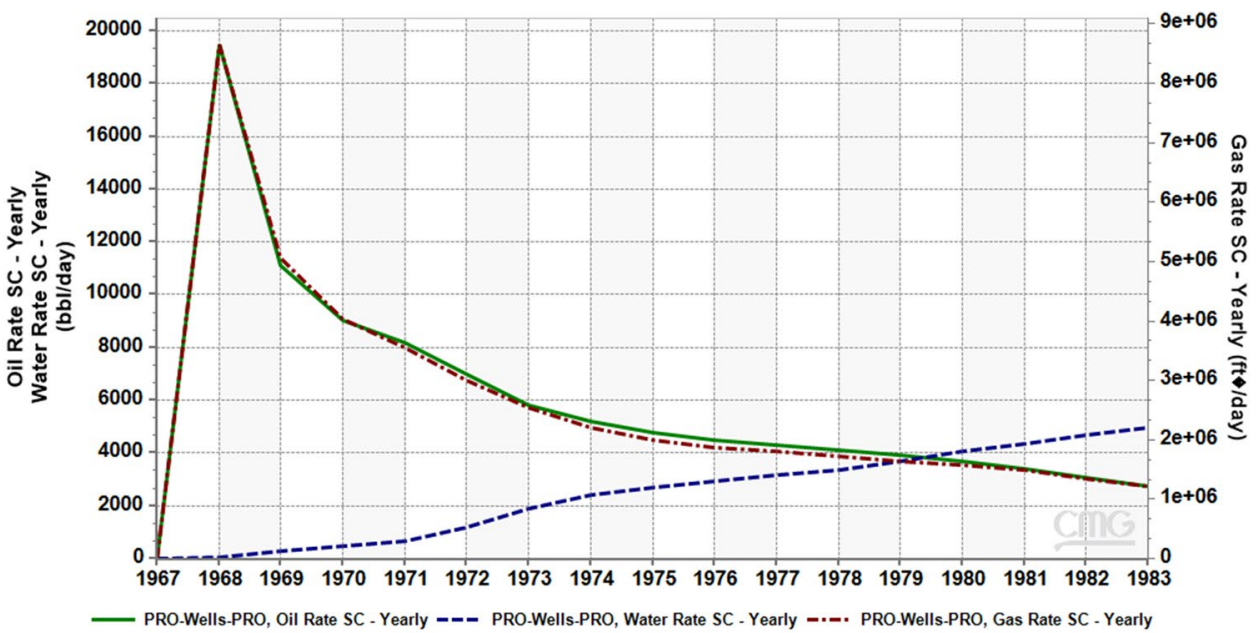

(b)

\section{Conclusion}

The methodology presented here has been grounded in a simple proxy function (see Eqs. 1-4) proposed in the literature, nevertheless, the results for both synthetic reservoir cases show it as a feasible alternative for the indication of a suitable location as a target position that exhibited a high productivity potential for oil recovery and for well placement appropriateness.

Unfortunately, a closer look to the cumulative oil production of individual wells placed by PP strategy have shown a drawback as some of them placed at a better ranked position, according to the methodology, had an inferior oil production performance than other wells placed at less ranked productivity potential. One possible fact for that seems to be related to a higher number of perforations in low water saturation blocks. Therefore, to try to overcome this drawback, it is recommended that further investigations of this strategy be addressed, especially, considering (1) alternative synthetic reservoirs, (2) how uncertainties on the parameters in Eq. 1 would affect the PP strategy, (3) modifying the proxy function to include additional important geometrical and/or petrophysical parameters that play a relevant role to oil recovery improvement, and last but not least, (4) perform a systematic comparative analysis between PP approach and alternative well placement techniques.

Acknowledgements WLR would like to thank Juan Mateo from CMG Brazil support for many helpful hints. The authors thank both, Petrobras and the Brazilian National Agency of Petroleum, Natural Gas and Biofuels (ANP) for the funding through the R\&D Project No. 2018/00051-8.

Funding Funding was provided by Petrobras (Grant No. 2018/00051-8). 


\section{Compliance with ethical standards}

Conflict of interest The authors declare that they have no conflict of interest.

Open Access This article is licensed under a Creative Commons Attribution 4.0 International License, which permits use, sharing, adaptation, distribution and reproduction in any medium or format, as long as you give appropriate credit to the original author(s) and the source, provide a link to the Creative Commons licence, and indicate if changes were made. The images or other third party material in this article are included in the article's Creative Commons licence, unless indicated otherwise in a credit line to the material. If material is not included in the article's Creative Commons licence and your intended use is not permitted by statutory regulation or exceeds the permitted use, you will need to obtain permission directly from the copyright holder. To view a copy of this licence, visit http://creativecommons.org/licenses/by/4.0/.

\section{References}

Avansi GD, Schiozer DJ (2015) UNISIM-I: synthetic Mmodel for reservoir development and management applications. Int J Model Simul Petrol Ind 09(01):21-30

Centro de Estudos de Petróleo - CEPETRO, State University of Campinas, São Paulo, Brazil. https://www.unisim.cepetro.unicamp.br/

CMOST AI User Manual (2019) CGM software suite. Computer Modelling GroupLtd., Calgary

Ding S, Jiang H, Li J, Tang G (2014) Optimization of well placement by combination of a modified particle swarm optimization algorithm and quality map method. Comput Geosci 18(05):747-762
Ding S, Lu R, Xi Y, Wang S, Wu Y (2019) Well placement optimization using direct mapping of productivity potential and threshold value of productivity potential management strategy. Comput Chem Eng 121:327-337

Fanchi JR (2010) Integrated reservoir asset management: principles and best practices. Elsevier, London

Floris FJT, Bush MD, Cuypers M, Roggero F, Syversveen A-R (2001) Methods for quantifying the uncertainty of production forecasts: a comparative study. Petrol Geosci 7:S87-S96

Guerra NY, Narayanasamy R (2005) Well location selection from multiple realisations of a geomodel using productivity potential maps - a heuristic technique. In: International oil conference and exhibition, Cancun, Mexico, SPE-102903-MS

Kharghoria A, Cakici M, Narayanasamy R, Kalita R, Sinha S, Jalali Y (2003) Productivity-based method for selection of reservoir drilling target and steering strategy, vol 01, p SPE-85341-MS. Presented at the SPE/IADC Middle East drilling technology conference and exhibition, Abu Dhabi, United Arab Emirates

Liu N, Jalali Y (2006) Closing the loop between reservoir modeling and well placement and positioning, vol 01, p SPE-98198-MS. Presented at the Intelligent Energy Conference and Exhibition, Amsterdam, The Netherlands

Min BH, Park C, Kang JM, Park HJ, Jang IS (2011) Optimal well placement based on artificial neural network incorporating the productivity potential. Energy Sources Part A Recov Util Environ Effects 33(18):1726-1738

Petroleum Engineering \& Rock Mechanics Group - PERM, Imperial College London, London, England. https://www.imperial.ac.uk/ earth-science/research/research-groups/perm/

Publisher's Note Springer Nature remains neutral with regard to jurisdictional claims in published maps and institutional affiliations. 